\title{
Verbreitung, Einstellung und Faktoren, welche die Meldehäufigkeit beeinflussen
}

\section{Cyrille Bocion, \\ Felix H. Sennhauser, \\ Bernhard Frey}

Interessenverbindungen: B. Frey ist Vorstandsmitglied der Schweizerischen Gesellschaft für Intensivmedizin.

Zur besseren Lesbarkeit wird bei Personen nur die männliche Form genannt. Es sind damit immer beide Geschlechter gemeint.
Korrespondenz:

PD Dr. med. Bernhard Frey Abteilung für Intensivmedizin und Neonatologie Universitäts-Kinderklinik Steinwiesstrasse 75 CH-8032 Zürich Tel. 0442667359 Fax 0442667168

Bernhard.Frey@kispi.unizh.ch

\section{Einleitung}

Kritische Zwischenfälle (critical incidents) sind ungewollte Ereignisse oder Ergebnisse, die den Patienten negativ beeinflussten oder beeinflussen könnten. Patienten, die eine Intensivpflege benötigen, weisen oft mehrere schwerwiegende medizinische Probleme auf, und es kommen besonders viele medizinische Geräte sowie potentiell gefährliche Medikamente zum Einsatz. Dies sind Gründe, warum Critical Incidents auf der Intensivstation möglicherweise häufiger sind als auf den allgemeinen Abteilungen [1]. Die Anästhesie und die Intensivmedizin haben als erste Disziplinen Zwischenfallmeldesysteme (Critical Incident Monitoring, CIM) als Instrument der Qualitätssicherung eingeführt [2-4]. Wir haben den Eindruck, dass auch in der Schweiz viele Intensivstationen diese Systeme einsetzen. Die einzelnen Systeme sind unterschiedlich, und es ist nicht bekannt, wie das CIM organisiert sein sollte, um möglichst qualitätsrelevant zu wirken.

Um von den bereits vorhandenen vielfältigen Erfahrungen mit dem Critical Incident Monitoring auf schweizerischen Intensivstationen (IPS) zu profitieren, führten wir eine Umfrage durch. Es interessierte uns, wie verbreitet das Critical Incident Monitoring ist, wie gross die Meldehäufigkeit ist und durch welche Faktoren sie beeinflusst wird und wie der formale Ablauf des Meldesystems organisiert ist. Weiter waren wir an konkreten Änderungen interessiert, die, resultierend aus berichteten Vorfällen, eingeführt worden sind. Wir untersuchten auch die Einstellung des Personals zu diesem Meldesystem und dessen Akzeptanz. Diese gesammelten Erfahrungen sollen zu Verbesserungen des Critical Incident Monitorings in den Intensivstationen der Schweiz beitragen und auch den andern medizinischen Disziplinen zugute kommen.

\section{Methode}

Wir erhielten für unser Projekt die Zustimmung und Unterstützung durch die Schweizerische Gesellschaft für Intensivmedizin (SGI). Im Sommer 2004 schickten wir den ärztlichen Leitern aller Intensivstationen der Schweiz einen zweiteiligen
Le Critical Incident Monitoring

dans les services de soins intensifs

suisses

Distribution, attitudes et facteurs, qui influencent la fréquence

\section{des nombres d'annonces}

Le Critical Incident Monitoring (CIM) est de plus en plus employé en médecine de soins intensifs comme moyen instrumental, afin d'assurer la qualité. Mais on ignore comment ce système devrait être organisé, afin qu'il ait autant que possible un effet significatif sur la qualité. A l'aide d'un sondage auprès de tous les services de soins intensifs suisses en été 2004, nous voulions profiter des expériences multiples déjà acquises avec le CIM. Le taux de réponse s'élevait à $69 \%$ (66 sur 96 services de réanimation). $65 \%$ des services ayant répondu, utilisent un CIM. La durée d'utilisation médiane est jusqu'ici de trois ans (variation 0,5-13). Le point de vue sur le CIM est en général positif, bien que $23 \%$ des utilisateurs mentionnent que le personnel se sent menacé par le procédé. Le personnel soignant recourt beaucoup plus souvent au système que les médecins. Au Tessin et en Suisse Romande, il existe des forums entre les différents services, afin de discuter des incidents critiques. Les formulaires d'annonce ont une structure très variable et contiennent en moyenne neuf questions (variation 3-27). Un mois avant le sondage rentrèrent en moyenne quatre annonces d'incident critique dans les différents services de soins intensifs (variation 0-30, plus d'annonces dans les services de catégorie de formation $A$ ). Les facteurs suivants signalisent avoir un effet positif sur l'augmentation de la fréquence des nombres d'annonces: la garantie 
Fragebogen. Der erste Teil (19 Fragen) beinhaltete formale Aspekte bezüglich der Anwendung des Meldesystems auf der jeweiligen Intensivstation: Erfassung der Critical Incidents, Bearbeitung, Organisation und Information. Im zweiten Teil ging es um die Einstellung zum Critical Incident Monitoring. Wir baten die befragten Personen, den ausgefüllten Fragebogen zusammen mit einer Kopie des in Gebrauch stehenden Zwischenfallmeldeformulars zurückzuschicken. Die Befragung erfolgte anonym, in der Hoffnung, damit die Studienteilnahme zu erleichtern. Die beantworteten Fragebogen wurden danach in eine Exceltabelle eingetragen und in ein statistisches Programm importiert (SPSS 11.0 für Mac, Version 11.0.4), um eine Kategorisierung von Daten und die statistische Analyse vorzunehmen.

Bei den Fragen über die Einstellung zum CIM konnten die Teilnehmer einen Score von 1 bis 5 angeben (1: stark zustimmend, 5: stark ablehnend). Für die statistische Auswertung wurden drei Gruppen gebildet: Scores 1-2 (zustimmend), Score 3 (weiss nicht), Score 4-5 (ablehnend). Wir haben Stationen, die ein Meldesystem benutzen, mit solchen, die keines benutzen, verglichen. Ferner analysierten wir Unterschiede zwischen Sprachregionen (Deutschschweiz vs. Romandie/ Tessin) und Weiterbildungskategorien (durch die SGI anerkannte Kategorien A, B oder C bzw. fehlende Weiterbildungsanerkennung). Wir untersuchten, ob die Anzahl Meldungen im letzten Monat eine Abhängigkeit zeigt von Faktoren wie Anonymität, Meldeformat (elektronisch oder Papier) und primärem Empfänger der Meldun-

Abb. 1

Verteilung der CIM-Anwendungsdauer.

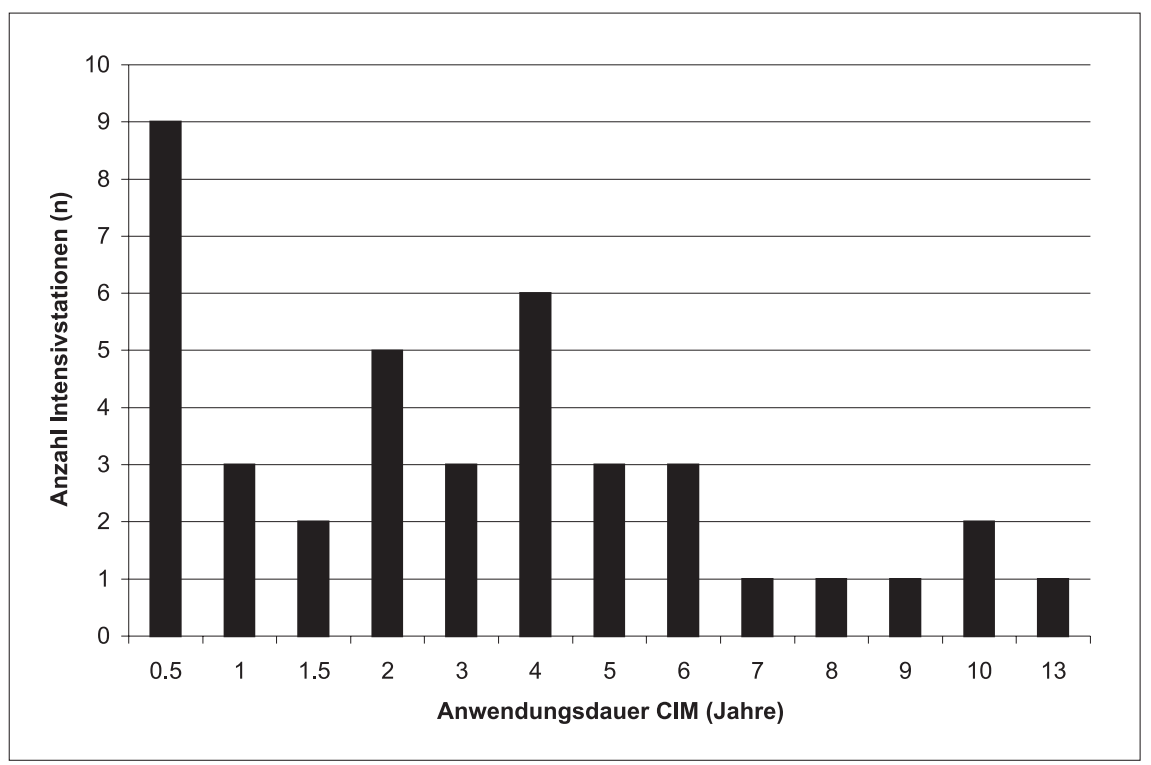

de I'anonymat (en ce qui concerne le collaborateur impliqué et la personne signalisant I'incident), l'acheminement primaire des annonces au groupe du CIM ou au responsable du CIM ( $p<0,001$, analyse univariate), I’organisation régulière de séances interdisciplinaires pour tous les collaborateurs afin de discuter des incidents $(p<0,01)$ et l'information du personnel absent $(p=0,06)$. Ces mesures pourraient augmenter la motivation à participer, diminuer les peurs et ainsi contribuer à l'annonce d'incidents critiques utiles pour la préservation de la qualité.

gen. Im Sinne einer Erfolgsanalyse des CIM in den verschiedenen Spitälern untersuchten wir die von den Beantwortenden angegebenen Massnahmen, die aufgrund von CIM-Meldungen eingeführt wurden. Auch die verschiedenen uns zur Verfügung gestellten Meldeformulare haben wir genauer unter die Lupe genommen: Anzahl Fragen, Freitext oder Auswahlantworten zum Ankreuzen und gewährleistete Anonymität (für die meldenden sowie am Zwischenfall beteiligten Personen).

\section{Statistik}

Die Resultate wurden in Form von Rohdaten dargestellt (Anzahl n). Damit ist auch sichtbar, dass die einzelnen Fragen nicht immer von allen beantwortet wurden: Die Nenner (n) der einzelnen Fragen sind unterschiedlich. Zur Darstellung der Meldehäufigkeit wurden die Intensivstationen in drei Gruppen eingeteilt: Stationen mit 0-5 Meldungen, 5-10 Meldungen und >10 Meldungen pro Monat. Die Berechnung des Einflusses von verschiedenen Faktoren auf die Meldehäufigkeit erfolgte mittels Chi-QuadratTest für Trends (Vergleich von mehreren in einer Ordnung stehenden Proportionen, univariate Analyse) sowie multipler stufenweiser Regression (multivariate Analyse). Die Signifikanz von Unterschieden zwischen Proportionen wurde mit dem Fisher's Exact Test berechnet. Ein $\mathrm{p}<0,05$ wurde als signifikant angenommen.

\section{Resultate}

Von 96 verschickten Fragebogen haben wir 66 zurückbekommen. Dies entspricht einer Rücklaufquote von 69\%. 43 (65\%) der antwortenden Abteilungen benutzen ein CIM. In bezug auf die CIM-Verbreitung konnten wir keinen Unterschied zwischen Deutschschweiz, Romandie und Tessin feststellen, wie auch nicht unter 
den verschiedenen IPS-Weiterbildungskategorien. 17 von 23 Abteilungen, die kein CIM benutzen, haben schon ernsthaft diskutiert, es einzuführen. Eine einzige Intensivstation hat wegen Spitalzusammenlegung mit dem CIM aufgehört. Die 66 Fragebogen wurden durch folgende Personen ausgefüllt: 49 durch den ärztlichen Leiter, 2 durch den Pflegeleiter, 7 durch den CIM-Verantwortlichen (Arzt), 3 durch den CIM-Verantwortlichen (Pflege), 2 durch den ärztlichen Leiter und den Pflegeleiter. In drei Fällen fand sich keine Angabe. Die mediane Anwendungsdauer von CIM beträgt 3 Jahre (Streu-

Abb. 2

Prozentuale Beteiligung von Ärzten und Pflegenden an den Critical-Incident-Meldungen.

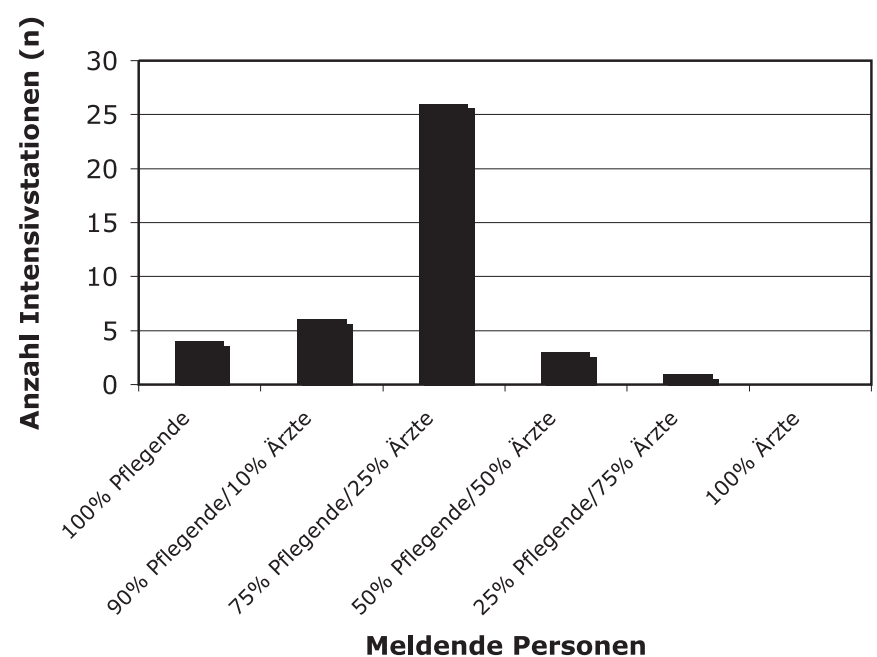

Abb. 3

Verteilung der Anzahl gemeldeter Zwischenfälle im letzten Monat.

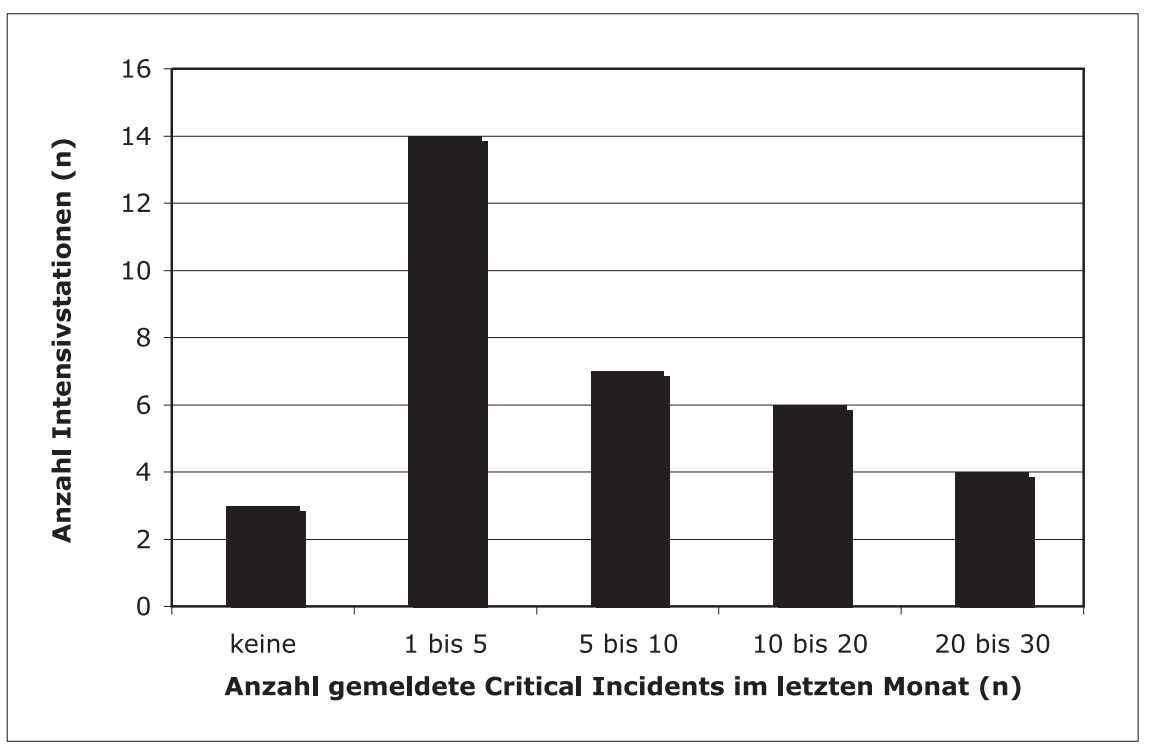

breite $0,5-13)$. Die detaillierte Verteilung ist in Abbildung 1 dargestellt. In Abbildung 2 sieht man die prozentuale Beteiligung von Ärzten und Pflegenden an den Critical-Incident-Meldungen nach Einschätzung der Fragebogenbeantworter. Man sieht eindeutig, dass das Instrument überwiegend von den Pflegenden benützt wird.

Eine Übersicht der strukturellen Organisation des CIM in den schweizerischen Intensivstationen wird in Tabelle 1 wiedergegeben. Zu ergänzen ist noch, dass 27 von 38 Antwortenden spitalübergreifende Foren (interne Foren) nützlich finden und dass 5 von 6 antwortenden Intensivstationen im Tessin oder in der Westschweiz an einem externen Forum teilnehmen (Besprechung von Zwischenfällen mit Intensivstationen ausserhalb des Spitals). Die Analyse erfolgt in fast allen Intensivstationen interdisziplinär (Ärzte und Pflege), in 13 Fällen durch den ärztlichen und pflegerischen Leiter, in den restlichen Fällen meist durch ein CIM- oder Q-Management-Team. In einem Spital nehmen noch die Materialverantwortliche und Pharmazeutin an der Analyse teil. Die eingegangenen Meldungen werden unterschiedlich oft analysiert: In 2 Intensivstationen zwei- bis viermal pro Monat, in 13 Intensivstationen einmal pro Monat, in 8 Intensivstationen alle 2 bis 3 Monate, in 5 Intensivstationen alle 4 bis 6 Monate und in 2 Intensivstationen nur einmal pro Jahr. In fünf Intensivstationen werden die Meldungen je nach Bedarf mehr oder weniger oft analysiert.

23 der 43 Abteilungen mit CIM schickten uns ihr Critical-Incident-Meldeformular. Die Formulare enthalten im Median 9 Fragen (Streubreite 3-27). Alle Fragebogen sind in vier mehr oder weniger weiter detaillierte Hauptteile gegliedert: Beschreibung, Ursache, Folgen und mögliche Prävention des Ereignisses. Diese Fragen können entweder durch Freitext oder Ankreuzen beantwortet werden. In Tabelle 2 ist dies zusammengefasst. In nur fünf Intensivstationen enthält das ganze Formular lediglich Freitextantworten. Die Anonymität der meldenden Person ist in 33 von 41 Fällen gewährleistet, die Anonymität des Patienten in 29 von 42 Fällen und die Anonymität der involvierten Mitarbeiter in 36 von 42 Fällen. 34 Antwortende gaben die Anzahl Zwischenfälle im letzten Monat an. Es ergibt sich ein medianer Wert von 4 (Streubreite 0-30) CriticalIncident-Meldungen (Abb. 3). Abbildung 4 zeigt den Zusammenhang zwischen der pro Monat aufgewendeten Zeit für die Analyse der Meldungen (Zusammenstellung und Auswertung, meist durch CIM-Verantwortliche) und der Anzahl eingegangener Meldungen im letzten Monat. Tabelle 3 zeigt, wer der erste Empfänger der 
Abb. 4

Zusammenhang zwischen der pro Monat aufgewendeten Zeit für die Analyse der Meldungen und der Anzahl gemeldeter Zwischenfälle im letzten Monat (aufgewendete Zeit = Anzahl beteiligte Personen der CIM-Gruppe $\times$ Stunden); $p=0,09, r=0,35$.

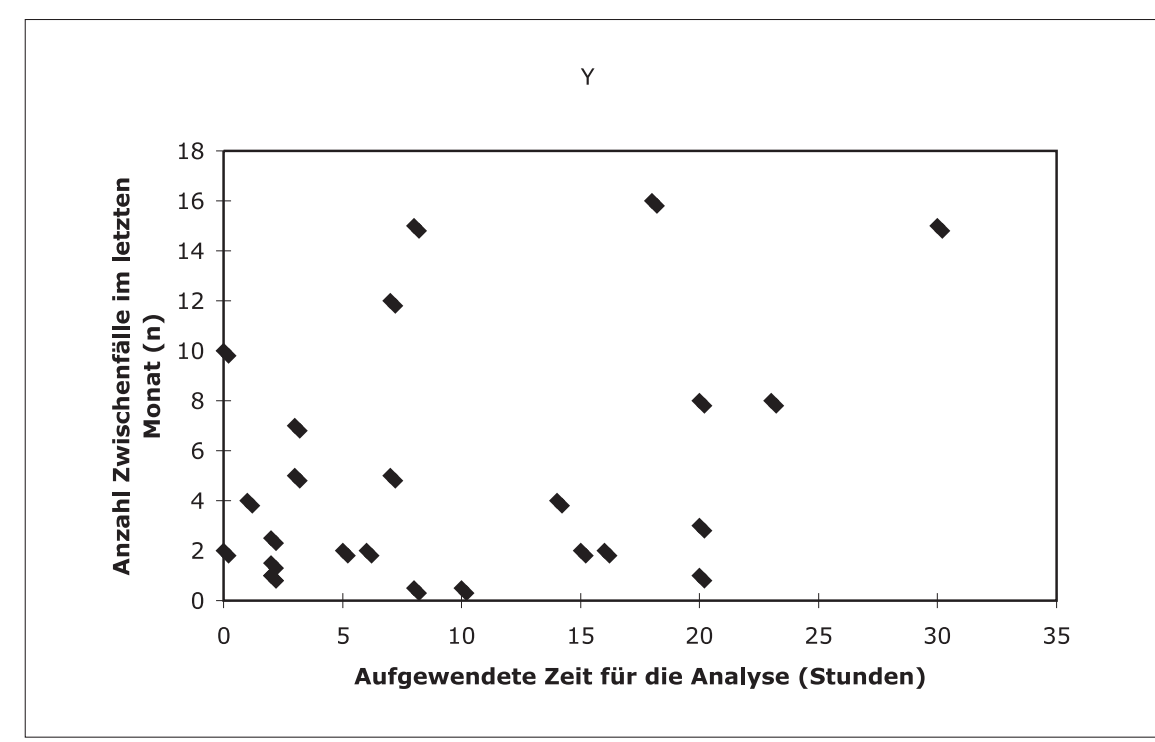

Tabelle 1

Details zum strukturellen Aufbau von CIM in schweizerischen Intensivstationen.

\begin{tabular}{|c|c|c|c|}
\hline Verantwortlich für CIM & Ja & Nein & Total \\
\hline Ein Arzt ist verantwortlich & $38^{*}$ & 4 & 42 \\
\hline Ein Pflegender ist verantwortlich & 36 & 6 & 42 \\
\hline \multicolumn{4}{|l|}{ Meldungen von CIM mit } \\
\hline Papierformular & 34 & 8 & 42 \\
\hline EDV-Formular & 26 & 16 & 42 \\
\hline Es gibt einen Briefkasten & 29 & 11 & 40 \\
\hline \multicolumn{4}{|l|}{ Methodische Einführung von CIM bei } \\
\hline Ärztlichem Personal & 36 & 5 & 41 \\
\hline Pflegendem Personal & 40 & 1 & 41 \\
\hline Paramedizinischem Personal & 18 & 23 & 41 \\
\hline Nichtmedizinischem Personal & 8 & 31 & 39 \\
\hline $\begin{array}{l}\text { Regelmässige Diskussionen über eingegangene Meldungen } \\
\text { für alle Mitarbeitenden finden statt }\end{array}$ & 29 & 12 & 41 \\
\hline $\begin{array}{l}\text { Internes Forum (relevante Zwischenfälle werden mit andern } \\
\text { Abteilungen des Spitals diskutiert) }\end{array}$ & 20 & 21 & 41 \\
\hline $\begin{array}{l}\text { Externes Forum (relevante Zwischenfälle werden mit Intensiv- } \\
\text { stationen ausserhalb des Spitals diskutiert) }\end{array}$ & 10 & 32 & 42 \\
\hline $\begin{array}{l}\text { Neben dem CIM gibt es noch andere Mechanismen, } \\
\text { um Zwischenfälle aufzugreifen }\end{array}$ & 24 & 17 & 41 \\
\hline Die CIM-Meldungen werden archiviert & 35 & 6 & 41 \\
\hline
\end{tabular}

Meldungen ist, in Abhängigkeit von der IPSWeiterbildungskategorie. In bezüglich Weiterbildungsmöglichkeiten höher eingestuften Intensivstationen kommen die Meldungen primär meistens zu einem speziellen CIM-Verantwortlichen, während sie in den andern (kleineren) Stationen eher auf dem Pult des Abteilungsleiters landen ( $\mathrm{p}=0,03$, Chi-Quadrat-Test für Trends).

Als Beispiele für andere Mechanismen als CIM, um Zwischenfälle zu detektieren, wurde folgendes genannt: Checkliste für Patientenaustritte, Materialkontrolle, Abteilungsrapport, Sturzprotokoll, Meldungen von Haftpflichtfällen und Morbiditätskonferenz.

In 29 Intensivstationen (von 41 Antworten) werden regelmässige interdisziplinäre Sitzungen abgehalten, um über die Zwischenfälle zu diskutieren (Tab. 1). In 69\% der Fälle werden Protokolle geführt und 76\% dieser Protokolle zirkulieren. In $75 \%$ der Intensivstationen mit regelmässigen Sitzungen wird das abwesende Personal informiert, am häufigsten mittels Newsletter (86\%). In 81\% der Fälle wird während dieser Sitzungen über Massnahmen diskutiert. Über 90\% dieser Intensivstationen beauftragen die Mitarbeitenden mit konkreten Aufgaben, setzen Zeitlimiten für deren Ausführung und protokollieren den Stand der Aufträge.

In der Tabelle 4 sind verschiedene Faktoren aufgelistet, welche die Meldehäufigkeit von Critical Incidents beeinflussen könnten. Signifikant mehr Critical-Incident-Meldungen werden in Abteilungen verfasst, in denen die Meldungen zu einem CIM-Verantwortlichen (und nicht primär zum Abteilungsleiter) gelangen sowie regelmässige interdisziplinäre Besprechungen der eingegangenen Meldungen stattfinden. Die Anonymität (bezüglich meldender Person, involviertem Mitarbeiter und Patient) beeinflusst die Meldehäufigkeit nicht signifikant, es fällt aber auf, dass in keiner Intensivstation, in der die Anonymität der meldenden Person oder der involvierten Mitarbeiter nicht gewährleistet ist, mehr als zehn Meldungen im letzten Monat eingegangen sind (Tab. 4). Weiter gingen in lediglich je einer Intensivstation mehr als zehn Meldungen ein, wenn Meldungen auf Papier nicht möglich waren bzw. abwesende Mitarbeiter über die interdisziplinären Critical-Incident-Besprechungen nicht informiert wurden. Die univariaten signifikanten Zusammenhänge werden bei Berechnung der multiplen Regression relativiert (abhängige Variable: Anzahl gemeldeter Critical Incidents im letzten Monat, unabhängige Variablen: Weiterbildungskategorie, Anonymität, primärer Empfänger der Meldungen, Meldungen auf Papier, regelmässige Besprechungen und In- 
Tabelle 2

Aufbau der Meldeformulare: Was wird gefragt und wie können die Antworten eingegeben werden?

\begin{tabular}{|c|c|c|c|c|c|}
\hline Rubrik & Freitext & Ankreuzen & $\begin{array}{l}\text { Freitext und } \\
\text { Ankreuzen }\end{array}$ & $\begin{array}{l}\text { Entsprechende Rubrik } \\
\text { wird nicht gefragt }\end{array}$ & Total \\
\hline Beschreibung des Critical Incidents & $22^{*}$ & 0 & 1 & 0 & 23 \\
\hline Ursache des Critical Incidents & 2 & 16 & 1 & 4 & 23 \\
\hline $\begin{array}{l}\text { Folgen des Critical Incidents } \\
\text { für den Patienten }\end{array}$ & 9 & 6 & 0 & $8(35 \%)$ & 23 \\
\hline $\begin{array}{l}\text { Vorschlag zur Prävention } \\
\text { von ähnlichen Ereignissen }\end{array}$ & 13 & 3 & 0 & $7(30 \%)$ & 23 \\
\hline
\end{tabular}

* Anzahl Intensivstationen.

Tabelle 3

Primärer Empfänger der Meldungen in Abhängigkeit von der IPS-Weiterbildungskategorie.

\begin{tabular}{|c|c|c|c|c|c|}
\hline $\begin{array}{l}\text { Wohin kommen } \\
\text { die Meldungen }\end{array}$ & WB-Kategorie A & WB-Kategorie B & WB-Kategorie C & Keine WB-Kategorie & $\mathbf{p}$ \\
\hline $\begin{array}{l}\text { Zum Abteilungsleiter } \\
\text { (direkt oder } \\
\text { über Briefkasten) }\end{array}$ & $1 *$ & 1 & 7 & 5 & \\
\hline $\begin{array}{l}\text { Zum CIM-Verantwortlichen } \\
\text { (über Briefkasten) }\end{array}$ & 7 & 4 & 8 & 3 & \\
\hline
\end{tabular}

Tabelle 4

Einfluss verschiedener Faktoren auf die Meldehäufigkeit (Anzahl Meldungen im letzten Monat).

\begin{tabular}{|c|c|c|c|c|}
\hline & $<5$ Zwischenfälle & 5-10 Zwischenfälle & >10 Zwischenfälle & $\mathbf{p}$ \\
\hline Anonym bezüglich Patient & $11^{*}$ & 5 & 7 & \multirow[t]{2}{*}{ ns } \\
\hline Nicht anonym bezüglich Patient & 6 & 2 & 3 & \\
\hline $\begin{array}{l}\text { Anonym bezüglich des involvierten } \\
\text { Mitarbeiters }\end{array}$ & 14 & 6 & 10 & \multirow[t]{2}{*}{ ns } \\
\hline $\begin{array}{l}\text { Nicht anonym bezüglich des involvierten } \\
\text { Mitarbeiters }\end{array}$ & 3 & 1 & 0 & \\
\hline Anonym bezüglich meldender Person & 13 & 5 & 10 & \multirow[t]{2}{*}{ ns } \\
\hline $\begin{array}{l}\text { Nicht anonym bezüglich meldender } \\
\text { Person }\end{array}$ & 4 & 2 & 0 & \\
\hline Meldungen direkt zum Abteilungsleiter & 11 & 1 & 0 & \multirow[t]{2}{*}{$<0,001$} \\
\hline Meldungen zum CIM-Verantwortlichen & 6 & 5 & 9 & \\
\hline Meldungen auf Papier & 13 & 5 & 9 & \multirow[t]{2}{*}{ ns } \\
\hline Meldungen auf Papier nicht möglich & 4 & 2 & 1 & \\
\hline $\begin{array}{l}\text { Regelmässige Besprechungen der } \\
\text { gemeldeten Zwischenfälle unter } \\
\text { den Mitarbeitern }\end{array}$ & 6 & 6 & 9 & \multirow[t]{2}{*}{$<0,01$} \\
\hline Keine regelmässigen Besprechungen & 11 & 0 & 1 & \\
\hline $\begin{array}{l}\text { Information der abwesenden } \\
\text { Mitarbeiter }\end{array}$ & 7 & 5 & 7 & \multirow[t]{2}{*}{0,06} \\
\hline $\begin{array}{l}\text { Keine Information der abwesenden } \\
\text { Mitarbeiter }\end{array}$ & 7 & 1 & 1 & \\
\hline
\end{tabular}


Tabelle 5

Änderungen («system changes») aufgrund von Zwischenfallmeldungen.

\begin{tabular}{lc} 
Eingeführte Änderung & Anzahl Intensivstationen \\
\hline Verordnung Medikamente & 7 \\
\hline Bereitstellung Medikamente & 11 \\
Verabreichung Medikamente & 15 \\
Beschriftung Medikamente & 10 \\
Apparate (Beispiele: EKG, Reanimationstisch) & 5 \\
Beatmung & 10 (davon 3 Beatmungstubus) \\
Überwachungsblätter & 3 \\
Informatik & 3 \\
\hline Transport/Verlegung & 4 \\
Organisatorisches & 11 \\
Teaching & 3 \\
Diverses & 18 \\
\end{tabular}

\section{Tabelle 6}

Übersicht über die Einstellung der Befragten zum Critical Incident Monitoring.

\begin{tabular}{|c|c|c|c|}
\hline Einstellung zum CIM & $\begin{array}{l}\text { Zustimmend } \\
\text { (Score 1-2) }\end{array}$ & $\begin{array}{l}\text { weiss nicht } \\
\text { (Score 3) }\end{array}$ & $\begin{array}{l}\text { ablehnend } \\
\text { (Score 4-5) }\end{array}$ \\
\hline \multicolumn{4}{|l|}{ CIM ist reine Bürokratie } \\
\hline users & $2(5 \%)$ & 5 & 34 \\
\hline non-users & $0(0 \%)$ & 2 & 20 \\
\hline \multicolumn{4}{|c|}{ CIM stört die Beziehungen innerhalb des Personals } \\
\hline users & $1(2 \%)$ & 9 & 31 \\
\hline non-users & $0(0 \%)$ & 2 & 20 \\
\hline \multicolumn{4}{|c|}{ CIM ist gut für die Teambildung } \\
\hline users & $28(65 \%)$ & 11 & 2 \\
\hline non-users & $13(57 \%)$ & 7 & 2 \\
\hline \multicolumn{4}{|c|}{ Das Personal versteht das Verfahren } \\
\hline users & $35(81 \%)$ & 4 & 2 \\
\hline non-users & $19(83 \%)$ & 1 & 2 \\
\hline \multicolumn{4}{|c|}{ Das Personal schätzt das Verfahren } \\
\hline users & $28(65 \%)$ & 9 & 4 \\
\hline non-users & $12(52 \%)$ & 6 & 4 \\
\hline \multicolumn{4}{|c|}{ CIM trägt dazu bei, die Patientensicherheit zu verbessern } \\
\hline users & $39(91 \%)$ & 1 & 1 \\
\hline non-users & $19(83 \%)$ & 2 & 1 \\
\hline \multicolumn{4}{|c|}{ Das Personal fühlt sich durch das Verfahren bedroht* } \\
\hline users & $10(23 \%)$ & 8 & 23 \\
\hline non-users & $4(17 \%)$ & 7 & 11 \\
\hline \multicolumn{4}{|c|}{ CIM ist mit grossem Zeitaufwand verbunden und bringt fast keinen Vorteil } \\
\hline users & $4(9 \%)$ & 6 & 31 \\
\hline non-users & $1(4 \%)$ & 6 & 15 \\
\hline
\end{tabular}

formation abwesender Mitarbeiter). Einzig die Weiterbildungskategorie bleibt als Faktor zurück, welcher die Meldehäufigkeit beeinflusst.

Auf die Frage nach vier bedeutsamen Änderungen, die aus dem CIM der zwei letzten Jahre resultierten, kamen zahlreiche Antworten. Diese sind in Tabelle 5, geordnet nach Kategorien, aufgelistet. Die Anzahl von Änderungen aufgrund kritischer Vorfälle im letzten Jahr betrug 0 bei 1 Befragten, 1-5 bei 19 Befragten, $5-10$ bei 10 Befragten und $>10$ bei 4 Befragten.

$71 \%$ der Befragten finden eine nationale Datenbank sinnvoll, 29 von 43 (67\%), die ein Meldesystem benutzen, und 12 von 16 (75\%), die ohne CIM arbeiten. Die Einstellung der Mitarbeiter zum CIM ist in der Tabelle 6 aufgeführt. Es wird der Meinungsunterschied zwischen den Befragten, die ein CIM benutzen, und denjenigen ohne Meldesystem (non-users) verglichen. Wir können feststellen, dass die CIM-Benutzer eine ähnliche Einstellung zum Critical Incident Monitoring haben wie die Non-Users. Es fällt auf, dass $23 \%$ der Users der Meinung sind, dass sich das Personal durch das Verfahren bedroht fühlt. Bei diesen 10 «skeptischen» Intensivstationen werden die Zwischenfälle wie folgt gemeldet: 4 direkt zum Abteilungsleiter, 3 zum Briefkasten, 1 am Morgenrapport, 2 ohne Angabe. In 7 von 17 (41\%) Intensivstationen aus der Westschweiz oder dem Tessin fühlen sich die Mitarbeiter durch das Verfahren bedroht gegenüber 7 von 46 (15\%) Intensivstationen in der Deutschschweiz ( $p=0,006$, Fisher's Exact Test).

\section{Diskussion}

Die Rücklaufquote betrug 69\%. 65\% der antwortenden Intensivstationen benutzen ein CIM. Fest steht, dass das System viel zu wenig von den Ärzten in Anspruch genommen wird. In den grösseren Intensivstationen (Weiterbildungskategorie A) werden mehr Meldungen verfasst und diese kommen primär meist zu speziellen CIMVerantwortlichen. Es bestehen Hinweise, dass die Anonymität der Meldungen, der primäre Empfänger der Meldungen sowie die regelmässige Durchführung von interdisziplinären Sitzungen zur Besprechung der Zwischenfälle die Meldehäufigkeit beeinflussen könnten. Eine landesweite Datenbank wird von 71\% der Antwortenden gewünscht. Das Personal steht dem System positiv gegenüber, auch wenn sich bis zu $23 \%$ durch das Verfahren bedroht fühlen.

In einer ähnlichen Umfrage in Grossbritannien im Jahr 2001 betrug die Rücklaufquote 34\% von 256 angeschriebenen Intensivstationen und 71\% der Antwortenden betrieben ein CIM [5]. Es ist in unserer Umfrage als positives Signal zu wer- 
ten, dass nur eine Intensivstation mit CIM aufgehört hat und dass nur sechs Intensivstationen, die kein CIM benutzen, noch nicht vorbereitend diskutiert haben, es einzuführen. Als Hauptgründe der noch nicht stattgefundenen Einführung werden der Widerstand des Pflegepersonals, Mangel an Zeit und finanziellen Mitteln, rechtliche Unklarheiten oder ein bezüglich Einstellungen noch nicht vorbereitetes Team genannt. Es war nicht möglich, die Non-Responders zu charakterisieren, da die Umfrage anonym verlief. Wenn wir aber vorsichtig annehmen, dass die Intensivstationen, die nicht geantwortet haben, kein CIM benutzen $(\mathrm{n}=30)$, stellen wir fest, dass mindestens $45 \%$ der Intensivstationen schweizweit über ein CIM-System verfügen. Es gibt keine zuverlässigen Angaben über die Verbreitung von CIM in andern Fachdisziplinen in der Schweiz. Hinweise ergeben sich aber aus einer Umfrage bei den Assistenzärztinnen und -ärzten der Schweiz im Jahre 2004 [6]. Auf die Aussage «An unserer Weiterbildungsstätte gibt es ein CIRS (Critical Incident Reporting System) oder ein anderes System zur Meldung kritischer Ereignisse und Komplikationen» antworteten folgende Prozentzahlen mit ja: Kinder und Jugendmedizin 71\%, Anästhesie $61 \%$, Chirurgie 49\%, Gynäkologie 47\%, Innere Medizin 41\%, Orthopädie $40 \%$, Psychiatrie $27 \%$.

Es geht aus der Umfrage klar hervor, dass die Critical-Incident-Meldungen hauptsächlich vom Pflegepersonal kommen und dass das System zu wenig von der Ärzteschaft gebraucht wird. Aus einer Befragung von 200 Ärzten und Pflegenden eines grossen Kinderspitals in den USA geht ebenfalls hervor, dass Pflegende ihre eigenen Fehler signifikant häufiger melden als Ärzte (OR 2.8) [7]. Zwei weitere Studien kommen zum gleichen Resultat $[8,9]$. Am Kinderspital in Melbourne, Australien, ergab eine Analyse des CIM, dass durch die schlechte Beteiligung der Ärzte Critical Incidents im Zusammenhang mit Fehldiagnosen oder verspäteter Therapie kaum erfasst werden [10]. Die Einführung und damit Verankerung des CIM bei den verschiedenen Berufsgruppen spielt somit eine wichtige Rolle für dessen Benutzung [6]. In diesem Zusammenhang ist es interessant, dass in unserer Umfrage lediglich eine Intensivstation keine Einführung für Pflegende anbietet, während dies für Ärzte bei fünf Stationen zutrifft (Tab. 1). Weiter wird in der Deutschschweiz in 10\% der antwortenden Intensivstationen das gesamte Personal eingeführt (Pflegepersonal, Ärzte, paramedizinisches Personal und nichtmedizinisches Personal), während es in der Westschweiz und im Tessin 46\% sind. Eine weitere Erklärung für den grossen Unterschied zwischen Beteiligung der Pflegenden und der Ärzte könnte darin liegen, dass das Pflegepersonal eher als Team auftritt, hingegen die Ärzte eher als Individuen agieren. Das erhaltene Zitat: «Pflege missbraucht CIRS-System für Beschwerden/Klagen; Ärzte nutzen das an und für sich gute System viel $\mathrm{zu}$ wenig» zeigt eine weitere negative Seite des CIM. Die Zusammenarbeit zwischen Pflegenden und Ärzten ist in der Intensivmedizin besonders eng. Damit sind eigentlich die Voraussetzungen ideal für einen konstruktiven gemeinsamen Umgang mit Fehlern. Das Thema sollte auch einen grösseren Stellenwert in der ärztlichen Aus-, Weiter- und Fortbildung haben [6].

Neben der involvierten Berufsgruppe suchten wir nach weiteren Faktoren, welche die Meldehäufigkeit beeinflussen können. Dazu korrelierten wir die Anzahl Meldungen im letzten Monat mit strukturellen Eigenschaften des CIM der betreffenden Intensivstationen. Die Anonymität, die Weiterleitung der Meldungen direkt an einen CIM-Verantwortlichen (und nicht zum Abteilungsleiter), die Meldung via Papierformular (gegenüber elektronischen Systemen), die Durchführung regelmässiger interdisziplinärer CIMMeetings sowie die Information abwesender Mitarbeiter könnten die Meldehäufigkeit positiv beeinflussen. Allerdings könnten die Zusammenhänge durch die Tatsache bedingt sein, dass die obigen Eigenschaften vor allem in grossen Intensivstationen (Weiterbildungskategorie A) mit vielen Meldungen ausgeprägt sind. Die Auswertungen erfolgen meist interdisziplinär (Ärzte und Pflege) und der zeitliche Aufwand richtet sich nach der Anzahl Meldungen ( $\mathrm{p}=0,09)$ (Abb. 4). Es lassen sich, mit der erwähnten Vorsicht, aus diesen Resultaten mögliche Strategien ableiten, um die Meldehäufigkeit in schweizerischen Intensivstationen zu erhöhen: Das Meldesystem sollte nicht in traditionelle hierarchische Strukturen eingebunden sein, weil dadurch für Pflegende und Ärzte der Eindruck entstehen mag, es gehe nicht primär um Qualitätssicherung, sondern um Mitarbeiterbeurteilung. In diese Richtung weist auch der Befund, dass zehn Intensivstationen angaben, das Personal fühle sich durch das Verfahren bedroht und dass in mindestens der Hälfte dieser Abteilungen die CriticalIncident-Meldungen direkt auf dem Pult des Abteilungsleiters landen. In der Tat ist die Meldehäufigkeit stark abhängig vom Klima einer Abteilung (ob eine Kultur der Schuldzuweisung 
besteht oder nicht) [11, 12]. Die Anonymität der Meldungen und insbesondere der aktive Einbezug aller Mitarbeiter in Meldung und Auswertung (interdisziplinäre Sitzungen mit der Möglichkeit, sinnvolle Massnahmen vorzuschlagen) erhöhen die Meldehäufigkeit. Schon Beckmann, eine Pionierin des CIM in der Intensivmedizin, führte ein nichtbestrafendes, freiwilliges und anonymes Meldesystem ein, um möglichst viele Zwischenfallberichte zu bekommen [2]. In der Umfrage von Taylor et al. [7] wurden von Pflegenden und Ärzten folgende Massnahmen genannt, um die Meldehäufigkeit zu erhöhen: methodische Einführungen in das Meldesystem, regelmässiges Feedback über eingegangene Meldungen und über erfolgreiche «system changes» aufgrund von Meldungen sowie ein elektronisches Meldeformat. Letzteren Faktor konnten wir in unserer Umfrage nicht bestätigen. Eher zeigte sich ein Trend Richtung weniger Meldungen mit elektronischen Systemen.

Ein externes Forum im Sinne von regelmässigen Critical-Incident-Besprechungen zwischen Intensivstationen verschiedener Spitäler wird ausser in der Westschweiz und im Tessin sehr wenig gebraucht. Solche Foren wie auch eine nationale Datenbank könnten sich prophylaktisch positiv auswirken durch Bekanntmachung von seltenen Ereignissen und gegenseitigen Erfahrungsaustausch.

Bei den uns zugegangenen Meldeformularen stellten wir eine grosse Streubreite der Anzahl Fragen sowie verschiedene Kombinationen zwischen Freitext und Auswahlantworten fest. In der Umfrage in Grossbritannien war die mediane Anzahl Fragen auf den Meldeformularen mit 36 deutlich höher [5]. In bezug auf die Gestaltung des Formulars ist es sicher wichtig, einen idealen Kompromiss zwischen der Genauigkeit der Daten zum Zwischenfall und einer Benutzerfreundlichkeit zu finden. Umfangreiche, komplizierte Meldeformulare, obwohl vielleicht wissenschaftlich interessant, eignen sich unserer Meinung nach nicht, ein erfolgreiches, qualitätsorientiertes CIM zu betreiben. Es fiel uns auf, dass mehrere Intensivstationen weder nach den möglichen Ursachen des Critical Incidents noch nach Vorschlägen zur Prävention ähnlicher Zwischenfälle in der Zukunft fragen. Diese Rubriken scheinen uns wichtig, denn deren Beantwortung könnte die Mitarbeitenden indirekt motivieren, das System vermehrt in Anspruch zu nehmen und eigene Lösungsvorschläge einzubringen. Diese Fragen sollen signalisieren, dass die Mitarbeitenden nicht nur melden sollen, sondern dass ihre Meinung zum Zwischenfall auch wichtig ist und ernst genommen wird.

Die meisten «system changes», die aufgrund von Critical-Incident-Meldungen eingeführt wurden, betreffen die medikamentöse Therapie und die künstliche Beatmung. Diese Kategorien rangieren auch bei den Critical-Incident-Meldungen anderer Erhebungen an erster Stelle [4].

Zur grundsätzlichen Einstellung ergibt die Umfrage, dass die Mitarbeiter eine positive Meinung gegenüber dem System haben, obwohl man beachten muss, dass die meisten Formulare durch den Abteilungsleiter ausgefüllt wurden (49/64). Somit ist es fraglich, ob die Meinung der hauptsächlichen Benutzer, nämlich des Pflegepersonals, realistisch in diese Statistik miteingeflossen ist. Um die exakte Einstellung des Personals, welches das System hauptsächlich benutzt, festzustellen, hätten wir das Formular direkt an dieses verteilen sollen. $\mathrm{Zu}$ denken gibt die Tatsache, dass 23\% der Benutzer sich durch das Verfahren «bedroht» fühlen, in der Westschweiz/ im Tessin in gar $41 \%$. Erfreulich dagegen ist, dass nur 4 von 47 Intensivstationen in der Deutschschweiz und nur 1 von 16 in der Westschweiz und im Tessin der Meinung sind, dass das CIM mit grossem Zeitaufwand verbunden sei und fast keinen Vorteil bringe.

\section{Schlussfolgerung}

Critical Incident Monitoring ist in den Intensivstationen der Schweiz gut etabliert. Das Personal steht dem System grundsätzlich positiv gegenüber, auch wenn es noch ungenügend von Ärzten in Anspruch genommen wird. Es empfiehlt sich die Bildung von interdisziplinären Gruppen, die die Meldungen analysieren. Die Anonymität sollte gewährleistet bleiben. Fachliche Einführung des Personals, regelmässige CIM-Sitzungen und -Besprechungen sowie Information des abwesenden Personals müssen unbedingt stattfinden. Diese Massnahmen erhöhen die Motivation, bauen Ängste ab und steigern die Anzahl von relevanten Meldungen. 


\section{Literatur}

1 Wilson DG, McArtney RG, Newcombe RG, McArtney RJ, Gracie J, Kirk CR, et al. Medication errors in paediatric practice: insights from a continuous quality improvement approach. Eur J Pediatr 1998;157:769-74.

2 Beckmann U, Baldwin I, Hart GK, Runciman WB. The Australian incident monitoring study in intensive care: AIMS-ICU. An analysis of the first year of reporting. Anaesth Intens Care 1996; 24:320-9.

3 Buckley TA, Short TG, Rowbottom YM, Oh TE. Critical incident reporting in the intensive care unit. Anaesthesia 1997;52:403-9.

4 Frey B, Kehrer B, Losa M, Braun H, Berweger L, Micallef J et al. Comprehensive critical incident monitoring in an neonatal-pediatric intensive care unit: experience with the system approach. Intensive Care Med 2000;26:69-74.

5 Thomas AN, Pilkington CE, Greer R. Critical incident reporting in UK intensive care units: a postal survey. J Eval Clin Pract 2003;9:59-68.

6 Siegrist M, Orlow P, Giger M. Weiterbildung aus der Sicht der Assistenzärzte. Schweiz Ärztezeitung 2005;86:412-23.
7 Taylor JA, Brownstein D, Christakis DA, Blackburn S, Strandjord TP, Klein EJ, et al. Use of incident reports by physicians and nurses to document medical errors in pediatric patients. Pediatrics 2004;114:729-35.

8 Vincent C, Stanhope N, Crowley-Murphy M. Reasons for not reporting adverse incidents: an empirical study. J Eval Clin Pract 1999;5:13-21.

9 Lawton R, Parker D. Barriers to incident reporting in a health care system. Qual Saf Health Care $2002 ; 11: 15-8$.

10 Dunn KL, Reddy P, Moulden A, Bowes G. Medical record review of deaths, unexpected intensive care unit admissions, and clinician referrals: detection of adverse events and insight into the system. Arch Dis Child 2006;91:169-72.

11 Cohen MR. Why error reporting systems should be voluntary. Br Med J 2000;320:728-9.

12 Reason J. Human error: models and management. Br Med J 2000;320:768-70. 